\title{
QUÍMICA ORGÂNICA NO BRASIL: SUA CONTRIBUIÇÃO NA SÍNTESE TOTAL DE PRODUTOS NATURAIS DESDE A FUNDAÇÃO DA SBQ"
}

\author{
Mauricio M. Victor*a,b \\ aDepartamento de Química Orgânica, Instituto de Química, Universidade Federal da Bahia, 40170-115 Salvador - BA, Brasil \\ 'Instituto Nacional de Ciência e Tecnologia em Energia e Ambiente, Universidade Federal da Bahia, 40170-115 Salvador - BA, Brasil
}

Recebido em 02/04/2017; aceito em 15/05/2017

\begin{abstract}
ORGANIC CHEMISTRY IN BRAZIL: ITS CONTRIBUTION IN THE TOTAL SYNTHESIS OF NATURAL PRODUCTS SINCE THE BRAZILIAN CHEMICAL SOCIETY FOUNDATION. An overview of the achivements in the field of the total synthesis of natural products in Brazil in the last 40 years is presented. These contributions are described on different classes of natural products since the birth of Brazilian Chemical Society in 1977. It was intended to create a brief review showing the evolution and current state of the art of Organic Chemistry in Brazil, registering growth and maturity of this area among Brazilian researchers, as well as the contribution of Brazilian organic chemical community in the new fields of this science.
\end{abstract}

Keywords: SBQ; Brazilian Chemical Society; synthetic organic chemistry overview.

\section{INTRODUÇÃO}

A Sociedade Brasileira de Química (SBQ) e seus associados estão em um ano de júbilo. Fruto de um imenso esforço de inserção internacional promovido por suas diretorias nos últimos anos, nosso país acolherá a realização do $46^{\circ}$ Congresso Mundial de Química da IUPAC (União Internacional de Química Pura e Aplicada), que pela primeira vez será realizado na América do Sul, conjuntamente com a Reunião Anual da SBQ que comemora seus 40 anos de fundação. Com o tema "Sustentabilidade e Diversidade Através da Química", este evento histórico consolida a maturidade e capacidade de nossa comunidade, e representa a definitiva inserção da química brasileira no cenário mundial.

Com a responsabilidade de ter um olhar sobre a química orgânica desde a fundação da SBQ em 1977, mesmo ano da primeira edição da Química Nova, este artigo busca coletar informações que mostrem a evolução de sua qualidade, em sua diversidade de objetos de pesquisa e desenvolvimento, além de indicar as áreas na fronteira do conhecimento e dos novos desafios que se apresentam à química orgânica. Já há excelentes documentos que registram os aspectos históricos da gênese da área no Brasil, especialmente os que tratam da faceta "orgânica" do acordo bilateral CNPq-NAS (Conselho Nacional de Pesquisas e Desenvolvimento, do Brasil, e National Academy of Sciences, dos Estados Unidos) $)^{1-3}$. Também um levantamento detalhado sobre a produção da área no Brasil, bem como uma análise sobre o quadro evolutivo nos trabalhos apresentados à Reunião Anual da SBQ e nos BMOS (Brazilian Meetings on Organic Synthesis), já foi realizado na edição comemorativa aos 25 anos da $\mathrm{SBQ}^{4}$. Deste modo, de forma a evitar a sobreposição de análises e a descrição repetitiva dos fatos, o autor buscou o desafio de se afastar da visão anteriormente buscada pelos colegas e mostrar a evolução da capacidade dos pesquisadores nacionais sob outra ótica.

Focando a análise nos 40 anos da Química Orgânica Sintética no Brasil, este levantamento busca dar um panorama geral da evolução da área no Brasil, empregando exemplos de sínteses totais de produtos naturais em diferentes épocas, agrupando-as em diferentes classes, que demonstrem o aumento da capacidade, de maturidade e

*e-mail: mmvictor@ufba.br

"Este artigo é dedicado aos 40 anos da Sociedade Brasileira de Química da evolução da comunidade sintética nacional. Por fim, não podemos deixar de homenagear todos os "orgânicos" brasileiros que contribuíram nestes últimos 40 anos para a consolidação e reconhecimento de nossa área de pesquisa, e agradecer a todos aqueles que com seu esforço construíram, e ainda constroem, as belezas desta maravilhosa ciência, e que permitiram a escrita deste artigo.

\section{SÍNTESES TOTAIS NO BRASIL}

A síntese total de moléculas no Brasil está intimamente ligada ao desenvolvimento do acordo CNPq/NAS e à fundação da SBQ e da revista Química Nova em 1978. Apesar de haver exemplos de sínteses anteriores, como a do sativeno e do copacanfeno via ciclizações radicalares ${ }^{5}$, já em 1978, no segundo número do periódico, há o primeiro relato de um trabalho de investigação sobre uma síntese total $^{6}$. A síntese da $\beta$-metileno- $\chi$-spirolactona foi fruto das investigações iniciais na área de química orgânica do acordo binacional, e permitiu posteriormente a síntese total da bakkenolida $\mathrm{A}^{7}$ (Figura 1).

Como seria de se esperar, em função da enorme biodiversidade nacional, vários compostos biodisponíveis foram empregados como pool na síntese total de produtos naturais. Um belo exemplo destes primeiros esforços foi a síntese do triterpenoide bicíclico en$t$ - $(\alpha)$-polipodatetraeno ${ }^{8}$. O material de partida, o ácido copálico, foi obtido a partir do óleo de copaíba, de origem amazônica e de largo uso medicinal (Esquema 1). Nesta mesma época o (+)-citronelol, empregando química de ciclização intramolecular de carbenoide de uma $(\alpha)$-diazo- $(\beta)$-cetossulfona intermediária, foi transformada no feromônio (+)-grandisol ${ }^{9}$. Também o (-)-isopulegol, terpeno facilmente obtido a partir do citronelal, foi empregado como material de partida para a síntese da (-)-mintlactona ${ }^{10}$. A etapa chave da curta síntese foi o emprego de tri-acetato de tálio (TTA) como agente ciclizador. Açúcares também foram empregados como pool quiral. A síntese da herbarumina- ${ }^{11}$, uma fitotoxina isolada de fungos, teve como material de partida a $L$-arabinose. A etapa chave desta síntese foi um acoplamento intermolecular de Nozaki-Hiyama-Kishi.

As estratégias de sínteses totais também se desenvolveram sem o uso de fontes naturais de matérias-primas. Uma das classes importantes de compostos naturais são os feromônios, substâncias importantes no controle de insetos em culturas, e por isso foco de atenção dos químicos sintéticos brasileiros. De fato, devido a nossa 


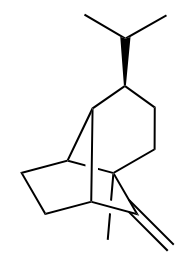

Sativeno (1976)

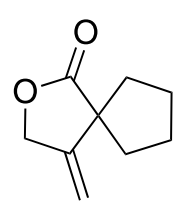

Spirolactona (1978)

Figura 1. Alvos sintéticos primordiais da química orgânica brasileira

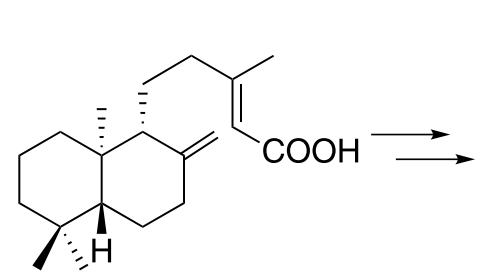

Ácido copálico<smiles>C=C1CC[C@H]2[C@@H](C)CCC[C@]2(C)[C@H]1CC(C)(C)C/C=C(/C)CC(C)C=C(C)C</smiles>

ent-( $\alpha)$-Polipodatetraeno (1995)<smiles>CC(C)C</smiles>

(+)-Citronelol

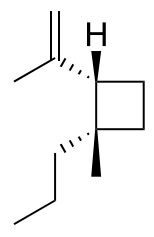

(+)-Grandisol (1996)<smiles>C=C(C)[C@@H]1CC[C@@H](C)C[C@H]1O</smiles>

(-)-Isopulegol
(-)-Mintlactona (2000)<smiles>O[C@H]1CO[C@@H](O)[C@@H](O)[C@H]1O</smiles>

$L$-Arabinose<smiles>CCC[C@H]1OC(=O)CCC/C=C\[C@@H](O)[C@@H]1O</smiles>

(+)-Herbarumina (2002)

Esquema 1. Sínteses totais baseadas em pool natural

pujante agroindústria, uma forte escola de pesquisa sobre feromônios se consolidou no Brasil. Um dos primeiros exemplos foi a preparação dos isômeros do propanoato do 8-metil-decan-2-ila, presentes no feromônio sexual da lagarta-da-raiz do milho (Diabrotica virgifera virgifera LeConte). Preparando o substrato inicial através de redução enzimática e tomando vantagem de posteriores alquilação e hidrogenação diastereosseletivas em anéis de tamanho médio, ambos isômeros $(2 S, 8 S)$ e $(2 S, 8 R)$ foram sintetizados ${ }^{12}$. Os compostos non-6-enoato de metila e o non-6-en-1-ol são componentes do feromônio de atração sexual da mosca-da-fruta do Mediterrâneo (Ceratitis capitata Wiedmann). Partindo de uma cicloexanona substituída e empregando uma reação de fragmentação de 2-cloro-1,3-dionas, o cloro-éster intermediário obtido foi transformado nos componentes do atrativo do inseto ${ }^{13}$. Há casos de um único componente que atua como feromônio. É o caso da maria-fedida ou pecervejo-da-soja (Nezara viridula Linnaeus). O sesquiterpeno bisaboleno de estereoquímica $(Z)-(1 S, 2 R, 4 S)$ foi sintetizado a partir do álcool perílico e empregou uma reação de olefinação ${ }^{14}$, bastante comum na preparação de insaturações em compostos atrativos de insetos. Outro inseto com componente único no feromônio atrativo que teve seu feromônio sintetizado foi o caruncho-do-fumo (Lasioderma serricorne Fabricius). A estratégia quimioenzimática empregada na preparação da serricornina ${ }^{15}$ envolveu o uso de fermento de padaria (S. cerevisiae). Há também uma vertente comercial na síntese de feromônios. Por exemplo, a preparação do feromônio de agregação da broca-do-olho-do-coqueiro (Rhynchophorus palmarum Linnaeus), que envolveu apenas uma etapa de adição de composto de Grignard a um aldeído ${ }^{16}$, permitiu a criação de um núcleo de estudos e uma incubadora dentro de uma universidade, de onde o composto é fornecido em escala. Por fim, como prova da evolução da comunidade brasileira nesta área, o feromônio do besouro-soldado (Chauliognathus fallax Germar), além de ter sido sintetizado, foi também identificado em nosso país ${ }^{17}$. Uso extensivo de métodos analíticos como eletroantenografia, cromatografia gasosa, espectrometria de massas e espectroscopia no infravermelho permitiu a identificação do (Z)-tricos-11-eno, que foi então sintetizado e confirmado como composto atrativo da espécie (Figura 2).

Outra classe de produtos naturais bastante explorada pelos sintéticos brasileiros são os alcaloides. Uma das primeiras estratégias empregadas na preparação destes compostos em solo nacional foi o emprego da reação de adição a íons $N$-acil-imínio seguida de uma adição de Michael sequencial (estratégia one-pot $)^{18}$. Apesar de permitir a síntese do produto natural apenas na forma racêmica, representou uma estratégia eficiente na preparação do alcaloide quinolizidínico lasubina II (Figura 3). Outra estratégia empregada nas primeiras sínteses totais foi a execução de sínteses formais, mas já com controle enantiosseletivo. Para a síntese formal do alcaloide indólico (+)-vincamina ${ }^{19}$, lançou-se mão do emprego de alquilações derracemizantes empregando iminas quirais, que permitiram a preparação de um intermediário em alto excesso enantiomérico (90\% ee, medida por RMN de ${ }^{1} \mathrm{H}$ com reagente quiral de deslocamento). A evolução nesta área pode ser sentida nos exemplos mais recentes da preparação de alcaloides. A policitrina A, por exemplo, foi preparada de maneira muito eficiente através do uso de reação de Heck-Matsuda ${ }^{20}$, que emprega tetra-flúor-boratos de aril-diazônios como substrato, e é reação em estudo e desenvolvimento em nosso país e de extenso uso em sínteses totais ${ }^{21}$. A síntese da (+/-)-preussina também exemplifica a evolução da química no Brasil. A preparação estereosseletiva da mesma, efetuada em apenas 3 etapas $^{22}$, teve como material de partida um diazo-oxo-alquilfosfonato desenvolvido em laboratório local2 ${ }^{23}$. Já a síntese da (-)-aporfina ${ }^{24}$ demonstra o domínio de reações de cicloadição na preparação de estruturas complexas. $\mathrm{O}$ 
<smiles>CCC(=O)O[C@@H](C)CCCCC[C@@H](C)CC</smiles>

Propanoato de $(2 S, 8 S)$-8-metil-decan-2-ila<smiles>CCC(=O)O[C@H](C)CCCCCC(C)CC</smiles>

Propanoato de $(2 S, 8 R)$-8-metil-decan-2-ila Diabrotica Virgifera (1990)<smiles>CCC(=O)C(C)C[C@@H](C)[C@H](O)CC</smiles>

$(4 S, 6 S, 7 S)$-Serricornina Lasioderma serricorne (1998)<smiles>[R]C/C=C\CCCC</smiles>

$\mathrm{R}=\mathrm{CO}_{2} \mathrm{Me}$

(E)-Non-6-enoato de metila $\mathrm{R}=\mathrm{CH}_{2} \mathrm{OH}$

(E)-Non-6-en-1-ol Ceratitis lapitata (1993)<smiles>C/C=C/C(O)CC(C)C</smiles>

(E)-(+/-)-6-Metil-hept-2-en-4-ol Rhynchophorus palmarum (2002)<smiles>CC(C)=CC/C=C(/C)[C@@H]1[CH][C@](C)(O)CC1</smiles>

(Z)-(1S,2R,4S)-Epóxi-bisaboleno Nezara viridula (1994)<smiles>CCCCC=CCC(C)C</smiles>

(Z)-Tricos-11-eno Chauliognathus fallax (2016)

Figura 2. Alguns feromônios sintetizados no Brasil<smiles>COc1ccc([C@@H]2C[C@H](O)C[C@@H]3CCCCN32)cc1OC</smiles>

Lasubina II (1993)<smiles>CC[C@]12CCCN3CCc4c(n(c5ccccc45)[C@](O)(C(=O)OC)C1)C32</smiles>

(+)-Vincamina (1999)<smiles>O=C1C(c2cc(Br)c(O)c(Br)c2)=C(c2cc(Br)c(O)c(Br)c2)C(=O)N1CCc1ccc(O)cc1</smiles>

Policitrina A (2013)<smiles>C[C@@H]1C[C@@H](O)[C@@H](Cc2ccccc2)N1C</smiles>

(+/-)-Preussina (2014)<smiles>CN1CCc2cccc3c2C1Cc1ccccc1-3</smiles>

(-)-Aporfina (2015)

Figura 3. Alguns alcaloides sintetizados no Brasil

esqueleto aporfínico foi construído através de uma cicloadição [4+2] entre o benzino e um derivado isoquinolínico, e a síntese foi finalizada através de resolução em pequena escala.

Dentre os alvos sintéticos escolhidos pela comunidade de orgânicos brasileiros, não poderia faltar a classe dos policetídeos ou oxigenados, onde a presença de hidroxilas, carbonilas e duplas ligações congregam uma grande diversidade de produtos naturais. Um dos exemplos iniciais da capacidade da comunidade sintética nacional em alcançar a construção destes compostos foi a (+/-)-pirenoforina. Empregando reação de alquilação de aldeído com ânion gerado a partir de um $\beta$-nitro-éster e reação de dimerização como etapas-chave ${ }^{25}$, a síntese racêmica deu início à demonstração da capacidade na preparação desta classe no Brasil. Aproximadamente na mesma época, o decanolídeo (+/-)-foracantolídeo I também foi preparado ${ }^{26}$. Uma clivagem oxidativa por nitrosação hidrolítica de um precursor bicíclico, metodologia desenvolvida no Brasil, permitiu a construção do anel carbônico que levou à preparação do alvo sintético. Outro decanolídeo preparado no Brasil que demonstrou a capacidade de construção dessa classe de compostos foi a (-)-decarestrictina $\mathrm{D}^{27}$. A reação de Nozaki-Hiyama-Kishi foi empregada na formação da ligação C-C da lactona, e o estudo da influência dos grupos protetores permitiu controle na formação de um novo centro estereogênico e a síntese enantiosseletiva do produto natural. Também a butirolactona (+)-blastmicinona foi preparada pelos "sintéticos" brasileiros. A síntese total do policetídeo envolveu a preparação de um organotelureto, seguida de sua resolução enzimática ${ }^{28}$. Esta estratégia permitiu a síntese com excessos enantioméricos superiores à $97 \%$. Contudo, o ápice da demonstração da capacidade sintética nacional veio com a preparação do (-)-marinisporolídeo $\mathrm{C}^{29}$, a maior e mais complexa molécula já sintetizada no Brasil (Figura 4). Acoplamento cruzado do tipo Stille, olefinações de Takai, Horner-Hadsworth-Emmons e de JuliaKocienski, reação de metátese, reações aldólicas tipo Mukaiyama, tipo Evans e com indução 1,5-anti, além de ampla manipulação de grupos protetores são exemplos de reações empregadas na preparação da complexa molécula, que consolidou de maneira definitiva a grande capacidade nacional neste campo da química orgânica.

\section{CONSIDERAÇÕES FINAIS}

A química orgânica sintética, como todos os ramos da ciência, está passando por profundas transformações. A busca por novas metodologias, praticidade, eficiência, escalabilidade e robustez é hoje centro e foco das pesquisas na área, além da associação com todas as premissas da sustentabilidade. ${ }^{30}$ Isso está obrigando a Química 
<smiles>C/C=C/C(=O)OC(C)CCC(=O)/C=C/C(=O)CCC(=O)OC(C)C</smiles>

(+/-)-Pirenoforina (1978)<smiles>CCCC[C@H]1C(=O)O[C@@H](C)[C@@H]1OC(=O)CC(C)C</smiles>

(+)-Blastmicinona (2010)<smiles>C[C@@H]1CCCCCCCCC1O</smiles>

(+/-)-Foracantolídeo I (1981)

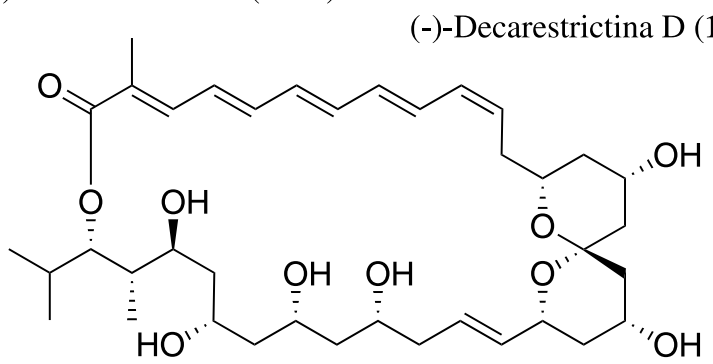

(-)-Marinisporolídeo C (2017)

Figura 4. Alguns compostos oxigenados sintetizados no Brasil

Orgânica, como todas as outras áreas, a se adaptar e buscar alternativas mais eficientes, mais limpas e mais elegantes para alcançar as sínteses totais de produtos naturais.

Nos últimos 40 anos é clara a evolução da comunidade sintética nacional. Nos anos 70, longas sínteses e alvos de menor complexidade eram a tônica. Poucas eram as contribuições nacionais, devido ao fato de termos uma incipiente comunidade sintética. Depois de 4 décadas, foi natural o amadurecimento. Alvos mais complexos, estratégias mais diretas e metodologias produzidas em nossos laboratórios já são comuns em várias publicações de autores brasileiros. Não nos parece mais ser possível afirmar que "tal substância" não pode ser sintetizada no Brasil. Os exemplos citados neste artigo são suficientes para demonstrar a capacidade que se instalou em nossa crescente comunidade, além de várias metodologias empregadas nas sínteses escolhidas terem sido desenvolvidas em nosso território.

Por fim, podemos afirmar que ganhamos maturidade para desenvolver e consolidar uma química orgânica sintética de reconhecimento internacional, e assim contribuir com a Ciência Brasileira e o desenvolvimento nacional.

\section{DIVISÃO DE QUÍMICA ORGÂNICA}

Número de filiados: 158 (atual)

\section{6-2018}

DIRETOR

VICE-DIRETOR

TESOUREIRO

2014-2016

DIRETOR

VICE-DIRETOR

TESOUREIRO

2012-2014

DIRETOR

VICE-DIRETOR

TESOUREIRO

2010-2012

DIRETOR

VICE-DIRETOR

TESOUREIRO
Mauricio Moraes Victor (UFBA)

Cristiano Raminelli (UNIFESP)

Giovanni Wilson Amarante (UFJF)

Rodrigo Octávio M. Alves de Souza (UFRJ) Antônio Carlos Bender Burtoloso (USP-SC) Cristiano Raminelli (UNIFESP)

Leandro Helgueira de Andrade (USP)

Paulo Henrique Schneider (UFRGS) Adilson Beatriz (UFMS)

Leandro Helgueira de Andrade (USP) Raquel Guimarães Jacob (UFPel) Mauricio Moraes Victor (UFBA)

\section{AGRADECIMENTOS}

O autor agradece à toda comunidade da Química Orgânica do Brasil, por todos seus diários esforços em prol de uma ciência mais digna e de melhor qualidade, e na busca por um país mais preparado para os novos desafios do desenvolvimento.

\section{REFERÊNCIAS}

1. Pilli, R. A.; Comasseto, J. V.; Ferreira, J. T. B.; Organic synthesis in Brazil: an overview: EDUSP, São Paulo, 1994.

2. Campbell, S.; Quim. Nova 2007, 30, 1393.

3. Brocksom, T. J.; Quim. Nova 2007, 30, 1394.

4. Correia, C. R. D.; Costa, P. R. R.; Ferreira, V. F.; Quim. Nova 2002, 25, 82.

5. Bakuzis, P; Campos, O. O. S.; Bakuzis, M. L. F.; J. Org. Chem. 1976, 41,3261 .

6. Petragnani, N.; Brocksom, T. J.; Ferraz, H. M. C.; Constantino, M. G.; Quim. Nova 1978, 2, 8.

7. Greene, A. E.; Deprès, J.-P.; Coelho, F.; Brocksom, T. J.; J. Org. Chem. 1985, 50, 3943.

8. Imamura, P. M.; Pantarotto, H.; Liebigs Ann. 1995, 1891.

9. Monteiro, H. J.; Zukerman-Schpector, J.; Tetrahedron 1996, 52, 3879.

10. Ferraz, H. M. C.; Grazini, M. V. A.; Ribeiro, C. M. R.; Brocksom, U.; Brocksom, T. J.; J. Org. Chem. 2000, 65, 2606.

11. Sabino, A. A.; Pilli, R. A.; Tetrahedron Lett. 2002, 43, 2819.

12. Ferreira, J. T. B.; Simonelli, F.; Tetrahedron 1990, 46, 6311.

13. Mahajan, J. R.; Tresvenzol, L. M. F.; J. Braz. Chem. Soc. 1993, 4, 179.

14. Baptistella, L. H. B.; Aleixo, A. M.; Liebigs Ann. 1994, 785

15. Pilli, R. A.; Riatto, V. B.; J. Braz. Chem. Soc. 1998, 9, 571.

16. Navarro, D. M. A. F.; Murta, M. M.; Duarte, A. G.; Lima, I. S.; Nascimento, R. R.; Sant'Ana, A. E. G. Quím. Nova 2002, 25, 32.

17. Vidal, D. M.; Fávaro, C. F.; Guimarães, M. M.; Zarbin, P. H. G.; J. Braz. Chem. Soc. 2016, 27, 1506.

18. Pilli, R. A.; Dias, L. C.; Maldaner, A. O.; Tetrahedron Lett. 1993, 34, 2729.

19. Alves, J. C. F.; Simas, A. B. C.; Costa, P. R. R.; Tetrahedron: Asymmetry 1999, 10, 297.

20. Canto, K.; Ribeiro, R. S.; Biajoli, A. F. P.; Correia, C. R. D.; Eur. J. Org. Chem. 2013, 8004.

21. Victor, M. M.; da Silva, G. S. B.; Quím. Nova 2016, 39, 859.

22. Rosset, I. G.; Dias, R. M. P.; Pinho, V. D.; Burtoloso, A. C. B.; J. Org. Chem. 2014, 79, 6748. 
23. Pinho, V. D.; Burtoloso, A. C. B.; J. Org. Chem. 2011, 76, 289.

24. Perecim, G. P.; Rodrigues, A.; Raminelli, C. Tetrahedron Lett. 2015, 56, 6848.

25. Bakuzis, P.; Bakuzis, M. L. F.; Weingartner, T. F.; Tetrahedron Lett. 1978, 27, 2371.

26. Mahajan, J. R.; de Araújo, H. C.; Synthesis 1981, 49.
27. Pilli, R. A.; Victor, M. M.; J. Braz. Chem. Soc. 2001, 12, 373.

28. Ferrarini, R. S.; Dos Santos, A. A.; Comasseto, J. V.; Tetrahedron Lett. 2010, 51, 6843.

29. Dias, L. C.; de Lucca, E. C., Jr.; J. Org. Chem. 2017, 82, 3019.

30. Brocksom, T. J.; Desiderá, A. L.; Alves, L. C.; de Oliveira, K. T.; Curr. Org. Synth. 2015, 12, 496. 